\title{
Imaging sand deformation at the grain scale
}

\author{
G. Viggiani ${ }^{1}$, P. Bésuelle ${ }^{1}$, S.A. Hall ${ }^{1}$, and J. Desrues ${ }^{1}$ \\ ${ }^{1}$ Laboratoire 3S-R, Université de Grenoble / CNRS, Grenoble, France
}

Shear banding, the localization of deformation into thin zones of intense shearing, is a phenomenon commonly observed in sand and other granular materials. The most valuable experimental contributions to the understanding of shear banding are those measuring, in one way or another, the full field of deformation in the specimen - which is the only means by which test results can be appropriately interpreted [1]. Full-field analysis of strain localization in sand possibly started in the late 1960s in Cambridge and was continued over the last decades in the work of a number of groups, including Grenoble. Most of these works were performed using specifically designed plane strain devices, and used a range of full-field methods, the more advanced of which allowed observation of the specimen throughout loading by optical methods, thereby permitting measurement of the evolving strain field. In the 1960s, x-ray radiography was first used to measure 2D strain fields in sand. From the early 1980s, x-ray tomography was used by a few groups working in geomechanics, including Desrues and coworkers (see [2] for a review). These studies provided valuable 3D information on localization patterning in sand, and also demonstrated the potential of x-ray tomography as a quantitative tool, e.g., for measuring the evolution of void ratio inside a shear band and its relation to critical state.

The recent advent of x-ray micro tomography, originally with synchrotron sources and now with laboratory scanners, has provided much finer spatial resolution, which opens up new possibilities for understanding the mechanics of granular media (in 3D) at the scale of the grain. For example, Oda and coworkers [3] presented micro tomography images of sand grains inside a shear band, showing organized structures that would not have been seen in standard x-ray tomography images (because of insufficient resolution) and that had only previously been observed in $2 \mathrm{D}$ thin sections.

It should be noted that the images by Oda were obtained post-mortem, i.e., after testing. However, a full understanding of the mechanisms of (localized) deformation can only be achieved if the entire deformation process is followed throughout a test while the specimen deforms. This is possible by using in-situ x-ray tomography (in-situ meaning x-ray scanning at the same time as loading). A number of such in-situ studies for triaxial tests on sand were performed over the last ten years or so, mostly using medical or industrial tomography systems, and in a few cases synchrotron micro tomography, which allows identifying individual sand grains and tracking their displacements throughout a test.

The aim of the present study is both to observe the material evolution under loading with grainscale resolution and to image the deformation processes. In recent work [4], we applied 3D Volumetric Digital Image Correlation (V-DIC) to a sequence of x-ray tomography images taken during a triaxial test on a clay-rock specimen. In the present paper, we show results of a similar DICbased analysis of deformation for sand specimens under triaxial compression. Two different granular materials were tested: Hostun sand, a fine-grained, angular siliceous sand with a mean grain size $\left(\mathrm{D}_{50}\right)$ of about $300 \mu \mathrm{m}$, and Caicos ooid, a material characterized by spheroidal grains with $\mathrm{D}_{50}$ of about $420 \mu \mathrm{m}$. In addition, we have developed a grain-scale V-DIC method that permits the characterization of the full kinematics (i.e., 3D displacements and rotations) of all the individual

This is an Open Access article distributed under the terms of the Creative Commons Attribution-Noncommercial License 3.0, which permits unrestricted use, distribution, and reproduction in any noncommercial medium, provided the original work is properly cited. 
grains in a specimen. So far, such a method has been applied only to Hostun sand. Preliminary results have been published in [5].

The tests were conducted using a specifically built in-situ setup that could be placed in the x-ray beam allowing the specimens to be scanned under load. The triaxial apparatus, made from Plexiglas (very transparent to $\mathrm{x}$-rays), is practically the same as a conventional system, except the much smaller size and the shape of the confining cell. As opposed to conventional triaxial systems, the tensile reaction force is carried by the cell walls and not by tie bars (which avoids having any obstacles to the x-rays). The axial load and hence the stress deviator are applied using a motor-driven screw actuator, which also does not interfere with the tomographic x-ray scans. See [4] for full details. Note that essentially the same setup was used in the experiments at the ESRF and at 3S-R.

Several triaxial compression tests were performed on dry specimens of Hostun sand and Caicos ooid. Results from just two tests are discussed herein, both performed under strain control at a confining pressure of $100 \mathrm{kPa}$. Both materials were tested starting from an initially dense packing, obtained by dry pluviation. Despite the relatively small sample size (height $22 \mathrm{~mm}$, diameter 11 $\mathrm{mm}$ ), the specimen can be considered large enough to be mechanically pertinent (i.e., its response can be considered representative of that of a larger mass of the material); in fact the sample comprises roughly 50000 grains for Hostun sand and 20000 for Caicos ooid. These reduced dimensions were imposed by the x-ray imager width at the ESRF, which was just $14 \mathrm{~mm}$ (the sample needed to be smaller than this to not risk passing out of the field of view). The spatial resolution (i.e., the voxel size) was set to $14 \times 14 \times 14 \mu \mathrm{m} 3$ at the ESRF, while it was slightly larger for the experiment on Caicos ooid performed in the scanner at $3 \mathrm{~S}-\mathrm{R}$ (where the side of the voxel was $14.7 \mu \mathrm{m}$ ). Such a resolution was enough to clearly identify the individual grains of both materials tested

The results obtained so far using the discrete V-DIC confirm the importance of grain rotations associated to strain localization. A clear correspondence can be established between the zones of the specimen experiencing localization of (continuum) shear strain and the zones where grain rotations are more intense. A deeper analysis of continuities/discontinuities at grain contacts and their evolution is now possible, and will be investigated in future work.

In constitutive modeling, one must understand the physics governing material behavior - from the micro scale to the continuum scale. This is particularly true when modeling emergent fine-scale mechanisms whose characteristic length scales are only a few particles wide, e.g., shear bands. With the tools we have presented in this paper, we now have the capability to capture experimentally and at a pertinent level of resolution the details of grain-scale processes, including those that underlie the localization phenomena of interest here. However, the potential of the approach has still to be fully exploited. Current and future directions of this research include more detailed analysis of: effects of the grains shape (e.g., angularity and aspect ratio of the grains); kinematics across grain contacts and its evolution with strain localization at the macro scale; emergence of grain-scale structures inside a shear band (e.g., the "columns" of aligned grains observed by Oda [3]); organized kinematics, in particular grain rotation, at the onset of shear banding and through its evolution.

\section{References}

1. G. Viggiani, Hall, S.A., Fourth Symposium on Deformation Characteristics of Geomaterials, Atlanta, USA (eds Burns, S.E., Mayne, P.W. \& Santamarina, J.C.), Amsterdam, IOS Press, 1, 3 26 (2008).

2. J. Desrues, 1st International Workshop on X-ray tomography for Geomaterials, Kumamoto, Japan, Balkema, 15-41 (2004).

3. M. Oda, T. Takemura, M. Takahashi, Géotechnique, 54, 539-542 (2004)

4. N. Lenoir, M. Bornert, J. Desrues, P. Bésuelle, G. Viggiani, Strain, 43, 193-205 (2007).

5. S.A. Hall, M. Bornert, J. Desrues, Y. Pannier, N. Lenoir, G. Viggiani, P. Bésuelle, Géotechnique, 59, in print (2010). 